\title{
EDITORIAL
}

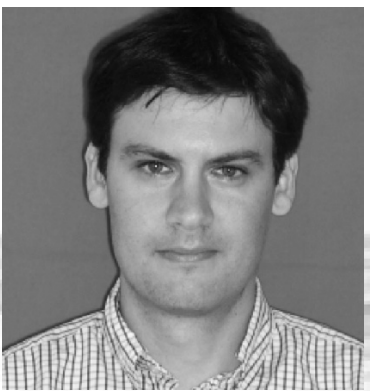

Donald Kossmann

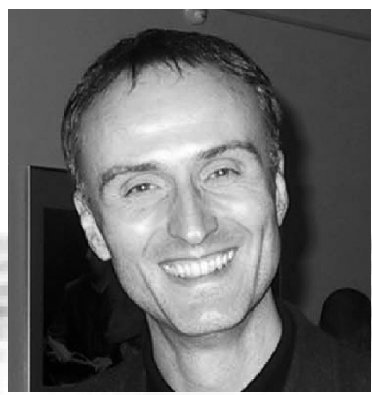

Frank Leymann

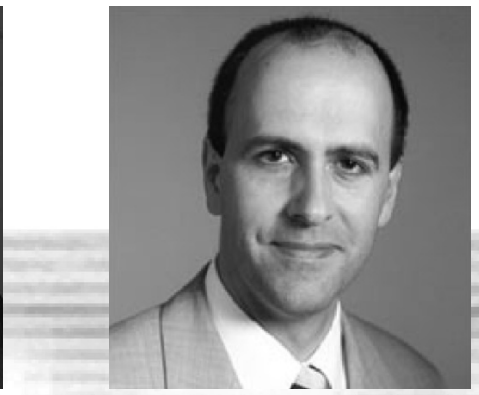

Dirk Taubner

\section{Web Services}

Web Services haben in den vergangenen Jahren geradezu einen Boom ausgelöst. Sowohl in der Industrie als auch an Hochschulen trifft diese Technologie auf breite Resonanz und sie berührt viele Disziplinen der Informatik (u.a. Verteilte Systeme, Programmiersprachen, Software Engineering, Informationssysteme, Betriebssysteme usw.). Ein Grund für das große Interesse an dieser Technologie kommt wohl auch daher, dass das magische Wort Web in ihrem Namen auftaucht. Tatsächlich haben Web Services nur nebenbei mit dem Web, wie die meisten es kennen, zu tun. Beim Web geht es um Menschen und wie Menschen Informationen publizieren und konsumieren. Bei Web Services geht es um Maschinen und wie Maschinen Informationen austauschen - und dies nicht notwendigerweise über das Web.

Neben Wortspielereien stehen hinter Web Services natürlich eine Reihe von technischen Entwicklungen. Das Ziel dieses Sonderheftes ist es, einige dieser technischen Entwicklungen vorzustellen und Anwendungen zu zeigen. Im ersten Artikel geben zwei der Gastherausgeber einen Überblick über die zentralen Web Services Standards, wie sie aufeinander aufbauen und welchen Einfluss sie auf die Erstellung von Anwendungen haben. Im zweiten Artikel von den Herren Brandner, Craes, Oellermann und Zimmermann wird der Einsatz von Web Services in einem industriellen Projekt aus dem Finanzsektor (deutsche Sparkassen) vorgestellt. Der Artikel erläutert die Anforderungen dieses Projektes, wieso und wofür Web Services eingesetzt wurden und die Erfahrungen, die mit dem Einsatz von Web Services gemacht wurden. Im dritten Artikel von den Herren Reinefeld und Schintke werden Grid Services erläutert. Diese Technologie kann als eine Erweiterung von Web Services angesehen werden, die zunächst auf die effizienten Nutzung von IT Ressourcen (Prozessoren, Speicher und Netzwerkbandbreite) in einem verteilten System fokussiert, aber auch als Erweiterung des Basis Web Service Modells angesehen werden kann. Besonders bekannt geworden sind Grid Services in jüngster Zeit im Zusammenhang mit der verteilten Auswertung von wissenschaftlichen Experimenten (z.B. in

"A Web service is a software system designed to support interoperable machine-tomachine interaction over a network. It has an interface described in a machine-processable format (specifically WSDL). Other systems interact with the Web service in a manner prescribed by its description using SOAPmessages, typically conveyed using HTTP with an XML serialization in conjunction with other Web-related standards."

H. Haas, A. Brown:
Web Services Glossary.
W3C Working Draft,
August 2003
der Physik und Genetik) in Rechenzentren quer durch Europa.

Die Idee zu diesem Themenheft entstand am Rande der sd\&m-Konferenz im Juli 2003. Über 1000 Kunden, Vertreter der Wissenschaft und Mitarbeiter haben sich dort zwei Tage dem Thema Web Services gewidmet. Es freut uns, dass jetzt mit diesem wichtigen Thema zur Integration und Koppelung von Systemen die Brücke zwischen Wissenschaft und Industrie in diesem Informatik-Spektrum geschlagen wird. 